\title{
Uncertain Internet Public Opinion Emergency Decision System Based on Case Reasoning and Grey Relational Analysis
}

\author{
Qiansheng Zhang ${ }^{*}{ }^{1}$, Bailin Xie ${ }^{2}$ and Xinmeng Zhang ${ }^{2}$ \\ ${ }^{I}$ School of Finance, Guangdong University of Foreign Studies, Guangzhou 510420, P.R. China; ${ }^{2}$ School of Informatics, \\ Guangdong University of Foreign Studies, Guangzhou 510420, P.R. China
}

\begin{abstract}
To facilitate uncertain internet opinion emergency decision involved with multiple fuzzy index values, we design a new prewarning decision system mechanism for uncertain internet opinion emergency based on case reasoning and grey relational analysis. Through the invented software in recent patents in data mining areas we first collect the internet opinion and detect the emergency data. Then by calculating the comprehensive grey relational coefficient between the unknown internet opinion emergency and each source emergency case, we can easily retrieve the optimal historical case for current emergency problem. And the related emergency managers may adopt or modify the corresponding warning decision solution of the most matched source case to make urgent decision for the new occurred internet public opinion emergency with uncertain indexes and incomplete weight information.
\end{abstract}

Keywords: Case reasoning, distance measure, grey relational coefficient, warning decision system.

\section{INTRODUCTION}

Internet public opinion emergency is a kind of the unconventional emergency induced by internet opinions. And internet public opinion emergency often involve a large number of uncertain risk factors. Moreover, when some internet opinion emergency happens, the emergency management departments hardly collect the complete emergency index value, instead they easily obtain the incomplete or uncertain index value of internet sentiment emergency which are usually represented by interval value, fuzzy value and vague value. Notably, it is a very important goal to forecast warning degree and make decision-making for uncertain internet opinion emergency in the government crisis management field. The early warning methods and emergency decision models have been studied by many researchers. For example, Zeng [1] proposed fuzzy hierarch analysis to determine the importance of early warning index and then proposed some related warning decision mechanism for internet opinion emergency. Lin [2] investigated internet opinion emergency warning decision system model based on fuzzy reasoning rule. $\mathrm{Li}[3]$ also presented grey warning method for internet opinion emergency. Some intelligent systems for internet public opinion emergency warning decision have been introduced in $[4,5]$. However, the above-mentioned emergency warning decision models are not very convenient to evaluate the risk alarm degree and make decision for uncertain internet public opinion emergency.

Recently, Huang [6] developed case-based reasoning method to study the enterprise crisis and early warning system. Patent US7813944 [7] provides a scoring system to

*Address correspondence to this author at the School of Finance, Guangdong University of Foreign Studies, Guangzhou, 510420, P.R. China; Tel: +86 39320862; Fax: +86 36 25408271; E-mail: zhqiansh01@126.com distinguish opinion characteristics and predict emergency. Patent US7945515 [8] invents a system for identification of emergency point of compromise. Zhong [9] proposed the case representation and reasoning method in emergency management decision system. Zhang [10] presented an emergency decision system based on case reasoning. However, most of existing emergency warning decision systems use the nearest neighbor algorithm based on similarity to retrieve the optimal warning decision case. They can not efficiently measure the similarity between uncertain internet opinion emergency cases with multiple fuzzy warning decision indexes.

With the increasing complexity of internet opinion emergency system and lack of the precise knowledge, the related emergency decision-maker usually provide his/her preference over emergency index with fuzzy linguistic assessment values, so in this paper we try to introduce a new internet opinion emergency warning decision system based on case reasoning and gray relational [11] retrieval algorithm. In this uncertain internet opinion emergency warning decision system, we aim to develop an improved grey relational analysis and case reasoning method for dealing with uncertain internet opinion emergency warning decision-making with fuzzy index value and incomplete weight information. By applying the grey relational analysis in internet opinion emergency case reasoning, we employ the comprehensive grey relational degree to calculate the matching degree between the current unknown internet opinion emergency and the stored source cases, and especially consider the different importance of warning decision index in the process of internet opinion emergency case reasoning. This system can exclude the influence of inferior index on warning decision. By the designed internet opinion emergency system we can easily obtain the proper warning degree and the corresponding scheme for the current internet opinion emergency problem. 


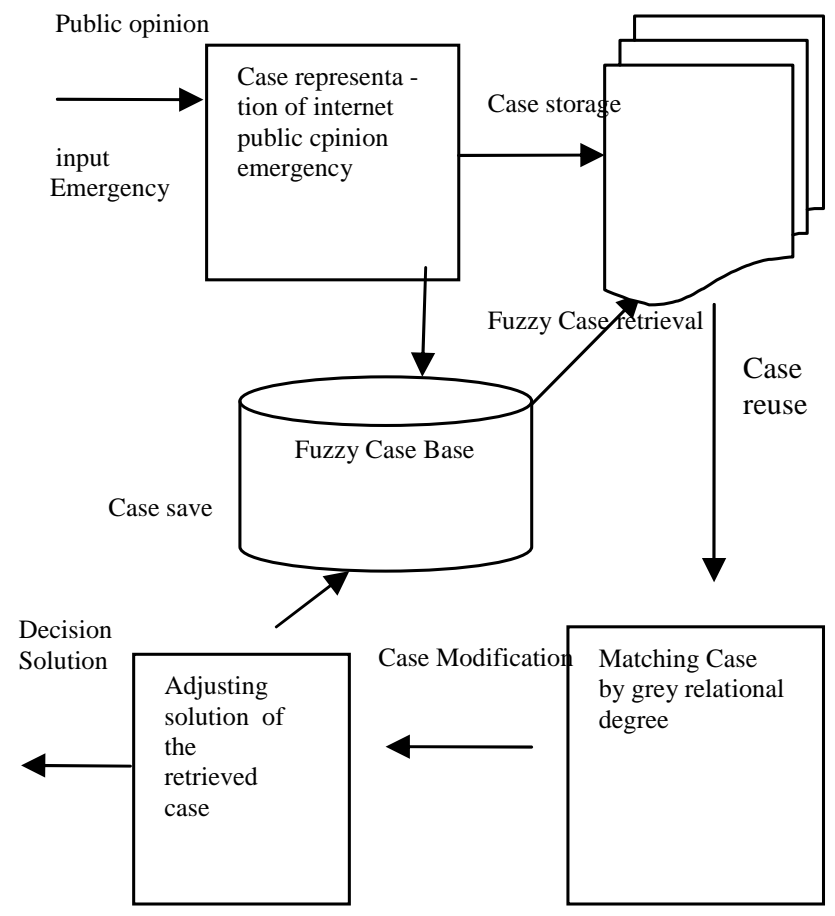

Fig. (1). Internet public opinion emergency warning decision system process.

\section{UNCERTAIN CASE REASONING MECHANISM FOR INTERNET PUBLIC OPINION EMERGENCY}

Case-based reasoning (CBR) is an important analogue reasoning methodology in recent artificial intelligence field. It has been successfully applied to many real-life decision problems like medical diagnosis, fault diagnosis, enterprise finance forecasting [6], risk management and emergency warning decision $[9,10]$. The case-based reasoning mechanism coincides with people's thinking habits and cognitive process. So, the CBR method is also suitable for early warning and urgent decision of uncertain internet opinion emergency. The core part of the warning decision support system is the design of case retrieval algorithm. Therefore, in this paper we design an effective warning decision system for uncertain internet opinion emergency based on grey relational retrieval method. First, we need to collect the related internet public opinion characteristics and emergency index data by using some software systems invented in Patents US8001597 [12], US7,953,695 [13], US7,895,139 [14], and US7,672,865 [15].

Through this prewarning decision system, we can retrieve the most matched source case of the current internet opinion emergency problem by searching all the historical emergencies in system case database. Then by modifying the matched source case, we get the optimal solution of current emergency problem and further update the new internet opinion case for storage backup. The entire case reasoningbased internet opinion emergency warning decision system structure is shown as in Fig. (1). It includes the procedure of case retrieval, case reuse, case modification and case study.
In the above emergency warning decision system process, we try to use case-based reasoning technique and grey relational analysis method to deal with uncertain internet public sentiment emergency warning decision problem with hybrid indexes. Generally, internet opinion emergency warning decision comprises the following three main steps:

Step 1. First, we can use multivariable statistical analysis method to determine the key warning decision indexes [16] and their weights, then properly represent the internet opinion emergencies as formalized case. Finally, we need to store the formalized internet opinion emergency cases as source cases in the warning decision system database and formalize current internet opinion emergency input.

Step 2. According to the above extracted important early warning decision indexes, we employ grey relational retrieval algorithm to compute the comprehensive grey relational degree between current internet opinion emergency and each source case, and retrieve the most similar historical emergency case to direct the current internet public opinion emergency warning decision.

Step 3. Due to the uncertain decision system environment, it is so difficult to retrieve the completely matched emergency source case for current unknown emergency. Therefore, we need to establish the related case study mechanism for internet public opinion emergency case modification and update. By adjusting the corresponding solution of the matched source case, we can obtain the optimal warning decision solution for the current internet opinion emergency. 
From the above process of internet public opinion emergency system case reasoning one can also see that most important step of all is to choose the effective case retrieval approach, which directly affects the timely internet opinion emergency warning decision. In traditional case-based reasoning system, researchers mainly utilized k-NN retrieval algorithm and induction index or knowledge index algorithm. Although k-NN algorithm is very simply and usefully in case reasoning, it only adapts to evaluate the similarity between emergency cases with crisp warning decision indexes, but hardly compute matching degree between uncertain emergency cases. However, the grey relational analysis method has been successfully applied in small size sample reasoning and fuzzy multi-attribute decision, and can forecast the divergence between the current case and historical case.

\section{INTERNET PUBLIC OPINION EMERGENCY CASE RETRIEVAL BASED ON GREY RELATIONAL COEFFICIENT}

Grey relational analysis [17] is an important theory method in uncertain grey system. In the developing of system, grey relational coefficient not only effectively compare the divergence between the current problem and old problem, but also measure the correlation coefficient between system cases and find the most matching cases of internet opinion emergency. If the grey relational degree between two cases is greater, then the trend of these two cases is more coincidence. So, the grey relational coefficient can reflect similarity between emergency cases with uncertain warning decision indexes.

In traditional case retrieval process, most researches use distance measures to calculate the similarity between internet opinion emergency indexes. However, the similarity based on these distances can reflect the position relation between two case data, but not reflect the emergency trend change. Thus, the traditional K-NN retrieval algorithm depending on similarity derived from these distances is not rational in uncertain emergency case reasoning system. So, in what follows we will use grey relation degree to replace traditional similarity measure to provide new case retrieval algorithm for internet opinion emergency warning decision system.

\subsection{Determine Weight of Each Warning Decision Index of Internet Public Opinion Emergency}

In the uncertain internet opinion emergency prewarning decision system based on case reasoning, the emergency knowledge and related expertise are usually represented by case structure. The characteristics of emergency case correspond to warning decision indexes.

Suppose $X=\left\{x_{1}, x_{2}, \cdots, x_{m}\right\}$ is the set of all $m$ relatively matching internet opinion emergency cases by using SQL sentence indexed by $n$ significant warning decision indexes $\left\{c_{1}, c_{2}, \cdots, c_{n}\right\}$. Then the set of all the uncertain internet opinion emergency cases can be formalized by the following decision matrix.

$$
X=\left[\begin{array}{lllll}
f_{1}(1) & f_{1}(2) & f_{1}(3) & \cdots & f_{1}(n) \\
f_{2}(1) & f_{2}(2) & f_{2}(3) & \cdots & f_{2}(n) \\
\cdots \cdots \cdots \cdots \cdots \cdots \cdots \cdots \cdots \cdots & \ldots \ldots \cdots \cdots \cdots \\
f_{m}(1) & x_{m}(2) & f_{m}(3) & \cdots & f_{m}(n)
\end{array}\right],
$$

where, $f_{i}(j)$ denote the value of internet opinion emergency $x_{i}$ with respect to the $j$ th warning decision index $c_{j}$, for $1 \leq j \leq n, 1 \leq i \leq m$.

As is well known, the weight of emergency characteristic index plays an important role in internet opinion emergency system reasoning and warning decision. And the weight of each index reflects the different importance in the process of case matching and emergency warning decision evaluation. Thus, in this paper we will take the following two methods to obtain the comprehensive weight of each warning decision index of internet public opinion emergency.

(1) First, by the related knowledge experience and expertise of internet opinion emergency, the emergency managers can compare any pair of warning decision indexes and assign the fuzzy preference degree over index pair to get fuzzy preference relation matrix $R=\left(r_{i j}\right)_{n \times n}$, where $r_{i j}$ denotes the fuzzy preference degree of index $c_{i}$ over index $c_{j}$. Then employing fuzzy AHP [18] we can get the first type of weight vector of all the emergency warning decision indexes as

$W^{(1)}=\left(w_{1}^{(1)}, w_{2}^{(1)}, \cdots, w_{n}^{(1)}\right)$,

where, $w_{j}^{(1)}$ is the weight of the jth warning decision index obtained by fuzzy AHP method.

(2) Second, according to the contribution rate of each index to the warning decision system result or from the discrimination ability between two emergency cases regarding this index, we can compute the second type of weight vector of warning decision indexes.

$$
W^{(2)}=\left(w_{1}^{(2)}, w_{2}^{(2)}, \cdots, w_{n}^{(2)}\right),
$$

where, for any $1 \leq j \leq n$,

$$
w_{j}^{(2)}=\sum_{i=1}^{m} \sum_{k=i+1}^{m} d\left(f_{i j}, f_{k j}\right)^{2} / \sum_{j=1}^{n}\left[\sum_{i=1}^{m} \sum_{k=i+1}^{m} d\left(f_{i j}, f_{k j}\right)^{2}\right] \text {, }
$$

$d\left(f_{i j}, f_{k j}\right)$ is the distance between the $j$ th index value of historical emergency cases $x_{i}$ and $x_{k}$.

Hence, we can combine the above two kinds of weight information to obtain the comprehensive weight vector of all the internet opinion emergency indexes.

$W=\left(w_{1}, w_{2}, \cdots, w_{n}\right) ;$

where, $w_{j}=w_{j}^{(1)} w_{j}^{(2)} / \sum_{j=1}^{n} w_{j}^{(1)} w_{j}^{(2)}, 1 \leq j \leq n$

Obviously, the comprehensive weight is more rational than each kind of weight above. 


\subsection{Grey Relational Analysis for Uncertain Internet Pub- lic Opinion Emergency Warning Decision System}

In the case-based reasoning process of internet opinion emergency system, each internet opinion emergency is formalized as case structure. The emergency criteria correspond to the warning decision indexes of internet public opinion emergency.

Suppose there are finite $m$ historical source emergency cases in the designed internet opinion emergency warning decision system. And each emergency is determined by $n$ early warning indexes, which can also be expressed by $x_{i}=\left(x_{i}(1), x_{i}(2), \cdots, x_{i}(n)\right) \quad(1 \leq i \leq m)$ with $n$ hybrid characteristic indexes in the internet opinion emergency warning decision system base. And we assume the new occurred internet opinion emergency is also described as $x_{*}=\left(x_{*}(1), x_{*}(2), \cdots, x_{*}(n)\right)$, where $x_{*}(j)$ is the $j$ th warning decision index value of internet opinion emergency. Retrieving the most matching source case is analogue to find the closest point near to the current emergency characteristic vector. Thus, we will adopt the grey relational theory to calculate the closeness of current internet opinion emergency with all the source cases in internet opinion emergency system.

First, we can compute the local grey relational degree of $j$ th index between the unknown internet sentiment emergency $x_{*}$ and the $i$ th source case $x_{i}(1 \leq i \leq m)$ below.

$$
\begin{aligned}
& \rho_{*_{i}}(j)=G\left(x_{*}(j), x_{i}(j)\right), 1 \leq j \leq n, \\
& =\frac{\min _{1 \leq j \leq n} d\left(x_{*}(j), x_{i}(j)\right)+\xi \max _{1 \leq j \leq n} d\left(x_{*}(j), x_{i}(j)\right)}{d\left(x_{*}(j), x_{i}(j)\right)+\xi \max _{1 \leq j \leq n} d\left(x_{*}(j), x_{i}(j)\right)}
\end{aligned}
$$

where, $d\left(x_{*}(j), x_{i}(j)\right)$ denotes the distance between current internet opinion emergency $x_{*}$ and source case $x_{i}$ with respect to jth characteristic warning decision index $c_{j}$; $\xi \in[0,1]$ is a given discriminate coefficient, in this paper we can take $\xi=0.5$ for convenience. Obviously, we can see that $0 \leq \rho_{*_{i}}(j) \leq 1$.

And the larger the value of $G\left(x_{*}(j), x_{i}(j)\right)$, the more similarity between the current internet opinion emergency $x_{*}$ and historical source case $x_{i}(1 \leq i \leq m)$ with respect to the warning decision index $c_{j}(1 \leq j \leq n)$.

Then we can construct the following grey relational coefficient matrix

$$
\rho=\left(\rho_{*_{i}}(j)\right)_{m \times n}=\left[\begin{array}{cc}
\rho_{*_{1}}(1) & \rho_{*_{1}}(2) \cdots \cdots \rho_{*_{1}}(n) \\
\rho_{*_{2}}(1) & \rho_{*_{2}}(2) \cdots \cdots \rho_{*_{2}}(n) \\
\rho_{*_{3}}(1) & \rho_{*_{3}}(2) \cdots \cdots \rho_{*_{3}}(n) \\
\cdots \ldots \ldots \ldots \ldots \ldots \ldots \ldots \ldots \ldots \\
\rho_{*_{m}}(1) & \rho_{*_{m}}(2) \cdots \cdots \rho_{*_{m}}(n)
\end{array}\right],
$$

where, $\rho_{i j}=\rho_{*_{i}}(j)$ denotes the grey relational degree between the current unknown internet opinion emergency $x_{*}$ and ${ }^{i}$ th source case ${ }^{x_{i}}$ regarding ${ }^{j}$ th decision index.

Thus, we can get the comprehensive grey relational coefficient between the current internet opinion emergency and each source case in the emergency system case base.

$$
\begin{aligned}
& \rho_{i}=G\left(x_{*}, x_{i}\right) \\
& =\sum_{j=1}^{n} w_{j} \rho_{*_{i}}(j)=\sum_{j=1}^{n} w_{j} G\left(x_{*}(j), x_{i}(j)\right)
\end{aligned}
$$

where, $w_{j}$ is the weight of ${ }^{j}$ th warning decision index obtained by formula (2). $\rho_{i}$ can be regarded as the comprehensive grey relational coefficient between the current internet sentiment emergency $x_{*}$ and historical source case $x_{i}$.

By using the grey relational coefficient $\rho_{i}$, we can get the corresponding similarity measure between emergency $x_{*}$ and source case $x_{i} . \forall \alpha \geq 1$,

$S_{*_{i}}=S\left(x_{*}, x_{i}\right)=1-\left[\sum_{j=1}^{n} w_{j}\left(1-\rho_{*_{i}}(j)\right)^{\alpha}\right]^{\frac{1}{\alpha}}$

From formula (5), one can see that the greater $\rho_{i}$ is, the more similarity between the current internet opinion emergency problem $x_{*}$ and source case $x_{i}$.

Therefore, we can retrieve the desirable source case according to the comprehensive grey relational degree $\rho_{i}$ between the current unknown emergency and each source case $x_{i} \quad(1 \leq i \leq m)$ in this designed internet opinion emergency warning decision system.

If $\rho_{s}=\max \left\{\rho_{i} / 1 \leq i \leq m\right\}$, then the most matching case of unknown internet opinion emergency is source case $x_{s}$. That is to say, the historical source case ${ }^{x_{s}}$ is most similar (close) to the current internet opinion emergency problem. Thus, we can use the corresponding warning decision solution of historical emergency case ${ }^{x_{s}}$ to facilitate the warning decision of the unexpected internet opinion emergency.

Due to the uncertain internet opinion emergency warning decision system environment, it is hard to find some source case completely matching the unknown emergency. And any warning decision solution of the matching case can only partially satisfy the current internet opinion emergency. So, we still need to modify the solution of the retrieval case to suit the current emergency. In the following, we can employ the distance measure to modify case and correct the warning decision solution for current unknown internet opinion emergency.

\section{CASE MODIFICATION FOR INTERNET PUBLIC OPINION EMERGENCY BASED ON DISTANCE}

Suppose the designed case base of internet opinion emergency warning decision system comprises $m$ historical source cases $\left\{x_{1}, x_{2}, \cdots, x_{m}\right\}$. We denote by the current un- 
known internet opinion emergency $x_{*}$. Assume the distance measure $d$ between the $j$ th warning decision index value of internet opinion emergencies $x_{*}$ and $x_{i}$ are given as follows.

(1) If the $j$ th index values of internet opinion emergency are crisp data, then the distance between them can be evaluated by

$$
d\left(x_{*_{j}}, x_{i j}\right)=\left|x_{*_{j}}-x_{i j}\right| /\left(\max _{j}-\min _{j}\right)
$$

where, $x_{*_{j}}, x_{i j}$ denote the $\mathrm{jth}$ index value of internet opinion emergencies $x_{*}$ and $x_{i}$, respectively; $\max _{j}, \min _{j}$ are the maximum and minimum value of $j$ th emergency index.

(2) If the $j$ th index value of internet opinion emergency $x_{*}$ and $x_{i}$ are interval values as $x_{*_{j}}=\left[x_{*_{j 1}}, x_{*_{j 2}}\right]$ $x_{i j}=\left[x_{i j 1}, x_{i j 2}\right]$ then the distance [19] between them is evaluated by

$d\left(x_{*_{j}}, x_{i j}\right)=\frac{\max \left\{\left|x_{*_{j 1}}-x_{i j 1}\right|,\left|x_{*_{j 2}}-x_{i j 2}\right|\right\}}{\left|\max \left\{x_{*_{j} 2}, x_{i j 2}\right\}-\min \left\{x_{*_{j} 1}, x_{i j 1}\right\}\right|}$

(3) If the $j$ th index values of internet opinion emergency are vague (intuitionistic fuzzy) values [20, 21], $x_{* j}=<\mu_{x_{*_{j}}}, v_{x_{*_{j}}}>x_{i j}=<\mu_{x_{i j}}, v_{x_{i j}}>$, then the distance between them is evaluated by

$d\left(x_{*_{j}}, x_{i j}\right)=\sqrt{\left[\left|\mu_{x_{*_{j}}}-\mu_{x_{i j}}\right|^{2}+\left|v_{x_{*_{j}}}-v_{x_{i j}}\right|^{2}+\left|\pi_{x_{*_{j}}}-\pi_{x_{i j}}\right|^{2}\right] / 2}$

(4) If the $j$ th index values of internet opinion emergency are fuzzy number $[22,23]$ as

$x_{*_{j}}=\left(x_{*_{j 1}}, x_{*_{j 2}}, x_{*_{j 3}}, x_{*_{j 4}}\right), x_{i j}=\left(x_{i j 1}, x_{i j 2}, x_{i j 3}, x_{i j 4}\right)$

then the distance between them is computed by

$d\left(x_{* j}, x_{i j}\right)$

$=1-\left[1-\frac{\sum_{k=1}^{4}\left|x_{*_{j k}}-x_{i j k}\right|}{4} \times \frac{\min \left(p\left(x_{*_{j}}\right), p\left(x_{i j}\right)+1\right.}{\max \left(p\left(x_{*_{j}}\right), p\left(x_{i j}\right)+1\right.}\right.$

where,

$$
\begin{aligned}
& p\left(x_{*_{j}}\right)=\sqrt{\left(x_{*_{j 1}}-x_{*_{j} 2}\right)^{2}+1}+\sqrt{\left(x_{*_{j 3}}-x_{*_{j} 4}\right)^{2}+1} \\
& +\left(x_{*_{j 3}}-x_{*_{j 2}}\right)+\left(x_{*_{j} 4}-x_{*_{j 1} 1}\right) ; \\
& p\left(x_{i j}\right)=\sqrt{\left(x_{i j 1}-x_{i j 2}\right)^{2}+1}+\sqrt{\left(x_{i j 3}-x_{i j 4}\right)^{2}+1} \\
& +\left(x_{i j 3}-x_{i j 2}\right)+\left(x_{i j 4}-x_{i j 1}\right) ;
\end{aligned}
$$

represent the perimeters of fuzzy numbers $x_{* j}$ and $x_{i j}$, respectively.

Notably, the weighted distance of current unknown internet opinion emergency $x_{*}$ to the $i$ th historical source case is defined as $d\left(x_{*}, x_{i}\right)=\sum_{j=1}^{n} w_{j} d\left(x_{*_{j}}, x_{i j}\right)$

From formula (10), we can see that the smaller the weighted distance $d\left(x_{*}, x_{i}\right)$ is, the nearer the source case $x_{i}$ close to the unexpected internet opinion emergency $x_{*}$.

If there are many source cases $x_{i}$ such that $d\left(x_{*}, x_{i}\right) \leq \delta$, where $\delta$ is the given referred distance threshold by the related emergency managers. Generally we take $\delta=0.1$ for convenience, so we can use all the solution of the relatively matching source cases $x_{i}$ to modify the warning decision solution of the most matching source case obtained by grey relational retrieval algorithm for the new occurred internet opinion emergency. This is the case modification mechanism by using the solutions of relatively similar source cases to adjust the solution of the most matching case for current internet opinion emergency.

\section{APPLICATION EXAMPLE}

Recently, grey relational analysis has been widely used in uncertain system control and decision and has drawn the attention of many researchers in order to perform multi-crit eria decision making [19-21]. In this section, we shall give an application example to demonstrate the feasibility of the proposed comprehensive grey relational analysis method to the internet opinion emergency system retrieval process with multiple uncertain warning decision indexes.

Example 1. Suppose we now designed an early warning decision system based on case reasoning for dealing with uncertain internet opinion emergency. And assume the case base of this emergency warning decision system stored six historical internet opinion emergency cases. And by using fuzzy AHP, we extract the following nine important indexes $\{$ News report, Micro-blog, Transmitting number, public sentiment spread speed, Public opinion tendency, Economic Loss, Casualty, Traffic jam, Response speed \} for internet opinion emergency warning decision. Moreover, each internet opinion emergence case is characterized and formalized by nine important warning decision index value as shown in Table 1. Now if there is an unexpected internet opinion emergency occurring in some area, the warning decision index values of the new occurred emergency are also recorded in Table $\mathbf{1}$, then we need to forecast the proper alarm degree of this new internet opinion emergency and retrieve the most matching historical case by this designed warning decision system to facilitate the urgent decision -making for the current unknown internet opinion emergency.

In the case base of warning decision system, we also evaluate the rang of news report number is [10, 5000]; the rang of micro-blog number is $[100,60000]$; the rang of transimitting number is [50, 40000]; and all the uncertain linguistic values in the warning decision system correspond to the following fuzzy numbers $[22,23]$ as in Table 2.

From the emergency case data in this warning decision system, we see that there are nine significant warning decision indexes, each internet opinion emergency case $x_{i}$ can be expressed by a multi-dimensional characteristic vector 
Table 1. Uncertain internet public opinion emergency warning decision system.

(1-1)

\begin{tabular}{|c|c|c|c|}
\hline Index & Case x1 & Case x2 & Case x3 \\
\hline \hline News report number & 2123 & 2680 & 1196 \\
\hline Micro-blog number & 87691 & 19903 & 5953 \\
\hline Transmitting number & 19768 & 12961 & Very quick \\
\hline public sentiment spread speed & Very slow & Medium & $<0.8,0.13>$ \\
\hline Public opinion tendency & $<0.6,0.3>$ & $<0.7,0.2>$ & {$[500,700]$} \\
\hline Economic Loss & {$[10,100]$} & {$[700,1000]$} & {$[50,70]$} \\
\hline Casualty number & {$[2,10]$} & {$[30,80]$} & Verere severe \\
\hline Traffic jam & Slight & quick & Very quick \\
\hline Response speed & Medium & & S \\
\hline
\end{tabular}

(1-2)

\begin{tabular}{|c|c|c|c|}
\hline Index & Case x4 & Case x5 & Case $x_{*}$ \\
\hline \hline News report number & 480 & 555 & 1534 \\
\hline Micro-blog number & 11247 & 8553 & 135678 \\
\hline Transmitting number & 2752 & 4258 & 5012 \\
\hline public sentiment spread speed & Extremely quick & Slow & $<0.8,0.2>$ \\
\hline Public opinion tendency & $<0.9,0.1>$ & $<0.5,0.4>$ & {$[600,800]$} \\
\hline Economic Loss & {$[200,900]$} & {$[4001000]$} & {$[40,60]$} \\
\hline Casualty number & {$[100,150]$} & {$[200,300]$} & Severe \\
\hline Traffic jam & Extremely severe & Medium & quick \\
\hline Response speed & Slow & Extremely quick & \\
\hline
\end{tabular}

$\left(f_{i 1}, f_{i 2}, \cdots, f_{i 9}\right), f_{i j}$ denotes the value of $j$ th index $c_{j}$ of internet opinion emergency $x_{i}$, for $1 \leq i \leq 5,1 \leq j \leq 9$.

By using Table 2, we can transfer all the uncertain fuzzy linguistic terms in the above early warning decision system to the corresponding Trapezoid fuzzy numbers.

\section{Table 2. Uncertain Linguistic Terms and The Corresponding} Fuzzy Numbers.

\begin{tabular}{|c|c|}
\hline Uncertain Linguistic Term & Trapezoid Fuzzy Number \\
\hline \hline $\begin{array}{c}\text { Extremely quick/Extremely high/ } \\
\text { Extremely severe }\end{array}$ & $(0.93,0.98,1.0,1.0)$ \\
\hline Very quick /Very high / Very severe & $(0.72,0.78,0.92,0.97)$ \\
\hline Quick/ High/ Severe & $(0.58,0.63,0.8,0.86)$ \\
\hline Medium & $(0.32,0.41,0.58,0.65)$ \\
\hline Slow / Low / Slight & $(0.17,0.22,0.36,0.42)$ \\
\hline Very Slow / Very Low / Very slight & $(0.04,0.1,0.18,0.23)$ \\
\hline $\begin{array}{c}\text { Extremely slow / Extremely Low / } \\
\text { Extremely slight }\end{array}$ & $(0,0,0.02,0.07)$ \\
\hline
\end{tabular}

Then, according to formulae (6)-(9) we first compute the following distance measures.

$$
\begin{aligned}
& d\left(f_{11}, f_{21}\right)=0.1116, d\left(f_{11}, f_{31}\right)=0.1858 \\
& d\left(f_{11}, f_{41}\right)=0.3293, d\left(f_{11}, f_{51}\right)=0.3142 \\
& d\left(f_{21}, f_{31}\right)=0.2974, d\left(f_{21}, f_{41}\right)=0.4409 \\
& d\left(f_{21}, f_{51}\right)=0.4259, \\
& d\left(f_{31}, f_{41}\right)=0.1435 d\left(f_{31}, f_{51}\right)=0.1285 \\
& d\left(f_{41}, f_{51}\right)=0.015 \\
& d\left(f_{12}, f_{22}\right)=0.3391, d\left(f_{12}, f_{32}\right)=0.3485 \\
& d\left(f_{12}, f_{42}\right)=0.3824, d\left(f_{12}, f_{52}\right)=0.3959 \\
& d\left(f_{22}, f_{32}\right)=0.6876, d\left(f_{22}, f_{42}\right)=0.0433 \\
& d\left(f_{22}, f_{52}\right)=0.0568, \\
& d\left(f_{32}, f_{42}\right)=0.7309 d\left(f_{32}, f_{52}\right)=0.7444 \\
& d\left(f_{42}, f_{52}\right)=0.0135 \\
& d\left(f_{13}, f_{23}\right)=0.0851, d\left(f_{13}, f_{33}\right)=0.1728 \\
& d\left(f_{13}, f_{43}\right)=0.2128, d\left(f_{13}, f_{53}\right)=0.194
\end{aligned}
$$




$$
\begin{aligned}
& d\left(f_{23}, f_{33}\right)=0.0877, d\left(f_{23}, f_{43}\right)=0.1277, \\
& d\left(f_{23}, f_{53}\right)=0.1089 \text {, } \\
& d\left(f_{33}, f_{43}\right)=0.04 d\left(f_{33}, f_{53}\right)=0.0212 \\
& d\left(f_{43}, f_{53}\right)=0.0188 \\
& d\left(f_{14}, f_{24}\right)=0.3956, d\left(f_{14}, f_{34}\right)=0.7203 \text {, } \\
& d\left(f_{14}, f_{44}\right)=0.8489, d\left(f_{14}, f_{54}\right)=0.1849 \\
& d\left(f_{24}, f_{34}\right)=0.3783, d\left(f_{24}, f_{44}\right)=0.5482 \text {, } \\
& d\left(f_{24}, f_{54}\right)=0.2235 \text {, } \\
& d\left(f_{34}, f_{44}\right)=0.2074 d\left(f_{34}, f_{54}\right)=0.555 \\
& d\left(f_{44}, f_{54}\right)=0.713 \\
& d\left(f_{15}, f_{25}\right)=0.1, d\left(f_{15}, f_{35}\right)=0.2 \text {, } \\
& d\left(f_{15}, f_{45}\right)=0.2646, d\left(f_{15}, f_{55}\right)=0.1 \\
& d\left(f_{25}, f_{35}\right)=0.1, d\left(f_{25}, f_{45}\right)=0.1732, d\left(f_{25}, f_{55}\right)=0.2 \text {, } \\
& d\left(f_{35}, f_{45}\right)=0.1 d\left(f_{35}, f_{55}\right)=0.3 d\left(f_{45}, f_{55}\right)=0.3606 \\
& d\left(f_{16}, f_{26}\right)=0.9091, d\left(f_{16}, f_{36}\right)=0.6061 \text {, } \\
& d\left(f_{16}, f_{46}\right)=0.8081, d\left(f_{16}, f_{56}\right)=0.9091 \\
& d\left(f_{26}, f_{36}\right)=0.303, d\left(f_{26}, f_{46}\right)=0.5051 \text {, } \\
& d\left(f_{26}, f_{56}\right)=0.303 \text {, } \\
& d\left(f_{36}, f_{46}\right)=0.303 d\left(f_{36}, f_{56}\right)=0.303 d\left(f_{46}, f_{56}\right)=0.202 \\
& d\left(f_{17}, f_{27}\right)=0.07, d\left(f_{17}, f_{37}\right)=0.06, d\left(f_{17}, f_{47}\right)=0.14 \text {, } \\
& d\left(f_{17}, f_{57}\right)=0.29 \\
& d\left(f_{27}, f_{37}\right)=0.02, d\left(f_{27}, f_{47}\right)=0.07, d\left(f_{27}, f_{57}\right)=0.22 \text {, } \\
& d\left(f_{37}, f_{47}\right)=0.08 d\left(f_{37}, f_{57}\right)=0.23 d\left(f_{47}, f_{57}\right)=0.15 \\
& d\left(f_{18}, f_{28}\right)=0.435, d\left(f_{18}, f_{38}\right)=0.555 \text {, } \\
& d\left(f_{18}, f_{48}\right)=0.713, d\left(f_{18}, f_{58}\right)=0.2235 \\
& d\left(f_{28}, f_{38}\right)=0.1451, d\left(f_{28}, f_{48}\right)=0.3375 \text {, } \\
& d\left(f_{28}, f_{58}\right)=0.2393 \text {, } \\
& d\left(f_{38}, f_{48}\right)=0.2074 d\left(f_{38}, f_{58}\right)=0.3783 \\
& d\left(f_{48}, f_{58}\right)=0.5482 \\
& d\left(f_{19}, f_{29}\right)=0.2393, d\left(f_{19}, f_{39}\right)=0.3783 \text {, } \\
& d\left(f_{19}, f_{49}\right)=0.2235, d\left(f_{19}, f_{59}\right)=0.5482 \\
& d\left(f_{29}, f_{39}\right)=0.1451, d\left(f_{29}, f_{49}\right)=0.435 \text {, } \\
& d\left(f_{29}, f_{59}\right)=0.3375 \text {, } \\
& d\left(f_{39}, f_{49}\right)=0.555 d\left(f_{39}, f_{59}\right)=0.2074 \\
& d\left(f_{49}, f_{59}\right)=0.713 \text {. }
\end{aligned}
$$

Using formula (1), for $1 \leq j \leq 9$,$$
w_{j}^{(2)}=\sum_{i=1}^{5} \sum_{k=i+1}^{5} d\left(f_{i j}, f_{k j}\right)^{2} / \sum_{j=1}^{9}\left[\sum_{i=1}^{5} \sum_{k=i+1}^{5} d\left(f_{i j}, f_{k j}\right)^{2}\right]
$$

we can get the second kind of weight vector of emergency warning decision indexes as

$W^{(2)}=\left(w_{1}^{(2)}, w_{2}^{(2)}, \cdots, w_{9}^{(2)}\right)$

$=(0.0835,0.1306,0.0626,0.1667,0.0663,0.1798,0.0464$, $0.132,0.132)$.

Given the fuzzy preference degree assessment from the related emergency experts and by employing fuzzy AHP method, we can easily get the first kind of weight vector of all the important warning decision indexes as below.

$W^{(1)}=\left(w_{1}^{(1)}, w_{2}^{(1)}, \cdots, w_{9}^{(1)}\right)$

$=(0.05,0.05,0.2,0.15,0.2,0.05,0.15,0.05,0.1)$

Thus, according to formula (2),

$$
w_{j}=w_{j}^{(1)} w_{j}^{(2)} / \sum_{j=1}^{9} w_{j}^{(1)} w_{j}^{(2)}, 1 \leq j \leq 9,
$$

we can combine the above two kinds of weight information and get the comprehensive weight vector of emergency warning decision indexes below.

$\mathrm{W}=(0.0429, \quad 0.0672, \quad 0.1288, \quad 0.2571, \quad 0.1364, \quad 0.0925$, $0.0716,0.0679,0.1357)$.

Further, with distance measure formulae (6)-(9) we can also calculate the following distances between the current unknown internet opinion emergency and each source case regarding nine warning decision indexes.

$$
\begin{aligned}
& d\left(x_{*}(1), x_{1}(1)\right)=0.118, d\left(x_{*}(2), x_{1}(2)\right)=0.2401, \\
& d\left(x_{*}(3), x_{1}(3)\right)=0.1846, d\left(x_{*}(4), x_{1}(4)\right)=0.6019, \\
& d\left(x_{*}(5), x_{1}(5)\right)=0.1732, d\left(x_{*}(6), x_{1}(6)\right)=0.7071, \\
& d\left(x_{*}(7), x_{1}(7)\right)=0.05, d\left(x_{*}(8), x_{1}(8)\right)=0.435, \\
& d\left(x_{*}(9), x_{1}(9)\right)=0, \\
& d\left(x_{*}(1), x_{2}(1)\right)=0.2297, d\left(x_{*}(2), x_{2}(2)\right)=0.5792, \\
& d\left(x_{*}(3), x_{2}(3)\right)=0.0994, d\left(x_{*}(4), x_{2}(4)\right)=0.2393, \\
& d\left(x_{*}(5), x_{2}(5)\right)=0.1, d\left(x_{*}(6), x_{2}(6)\right)=0.202, \\
& d\left(x_{*}(7), x_{2}(7)\right)=0.02, \\
& d\left(x_{*}(8), x_{2}(8)\right)=0, d\left(x_{*}(9), x_{2}(9)\right)=0.2393, \\
& d\left(x_{*}(1), x_{3}(1)\right)=0.0677, d\left(x_{*}(2), x_{3}(2)\right)=0.1084, \\
& d\left(x_{*}(3), x_{3}(3)\right)=0.0118, d\left(x_{*}(4), x_{3}(4)\right)=0.1451, \\
& d\left(x_{*}(5), x_{3}(5)\right)=0.1, d\left(x_{*}(6), x_{3}(6)\right)=0.101, \\
& d\left(x_{*}(7), x_{3}(7)\right)=0.01, \\
& d\left(x_{*}(8), x_{3}(8)\right)=0.1451, d\left(x_{*}(9), x_{3}(9)\right)=0.3783, \\
& d\left(x_{*}(1), x_{4}(1)\right)=0.2112, d\left(x_{*}(2), x_{4}(2)\right)=0.6225, \\
& d\left(x_{*}(3), x_{4}(3)\right)=0.0283, d\left(x_{*}(4), x_{4}(4)\right)=0.3375,
\end{aligned}
$$


$d\left(x_{*}(5), x_{4}(5)\right)=0.1, d\left(x_{*}(6), x_{4}(6)\right)=0.404$,

$d\left(x_{*}(7), x_{4}(7)\right)=0.09$,

$d\left(x_{*}(8), x_{4}(8)\right)=0.3375, d\left(x_{*}(9), x_{1}(9)\right)=0.207$,

$d\left(x_{*}(1), x_{5}(1)\right)=0.1962, d\left(x_{*}(2), x_{5}(2)\right)=0.6359$,

$d\left(x_{*}(3), x_{5}(3)\right)=0.0094, d\left(x_{*}(4), x_{5}(4)\right)=0.435$,

$d\left(x_{*}(5), x_{5}(5)\right)=0.2646, d\left(x_{*}(6), x_{5}(6)\right)=0.202$,

$d\left(x_{*}(7), x_{5}(7)\right)=0.24$,

$d\left(x_{*}(8), x_{5}(8)\right)=0.2393, d\left(x_{*}(9), x_{5}(9)\right)=0.5482$.

From formula (3), we can calculate the local grey relational degree between current emergency $x_{*}$ and source case $x_{i}$ regarding each warning decision index $c_{j}, 1 \leq i \leq 5$, $1 \leq j \leq 9$,

$\rho_{*_{i}}(j)=G\left(x_{*}(j), x_{i}(j)\right)$

$=\frac{\min _{1 \leq j \leq 9} d\left(x_{*}(j), x_{i}(j)\right)+\xi \max _{1 \leq j \leq 9} d\left(x_{*}(j), x_{i}(j)\right)}{d\left(x_{*}(j), x_{i}(j)\right)+\xi \max _{1 \leq j \leq 9} d\left(x_{*}(j), x_{i}(j)\right)}$,

Then we can construct the following grey relational matrix

$\rho=\left(\rho_{*_{i}}(j)\right)_{5 \times 9}=$

$\left[\begin{array}{lllllllll}0.7498 & 0.5956 & 0.657 & 0.37000 & 0.6712 & 0.3333 & 0.8761 & 0.44840 & 1.0000\end{array}\right]$

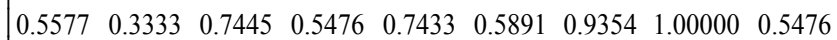

$\begin{array}{lllllllll}0.7754 & 0.6693 & 0.9910 & 0.5958 & 0.6887 & 0.6864 & 1.0000 & 0.59580 & 0.3510\end{array}$ $\begin{array}{lllllllll}0.6499 & 0.3636 & 1.0000 & 0.5234 & 0.8257 & 0.4747 & 0.8462 & 0.52340 & 0.6552\end{array}$ $\begin{array}{lllllllll}0.6429 & 0.3493 & 1.0000 & 0.4414 & 0.5686 & 0.6359 & 0.5933 & 0.59400 & 0.3843\end{array}$

According to formula (4),

$\rho_{i}=\sum_{j=1}^{9} w_{j} \rho_{*_{i}}(j)=\sum_{j=1}^{9} w_{j} G\left(x_{*}(j), x_{i}(j)\right)$,

we immediately obtain the comprehensive grey relational coefficient below.

$\rho_{1}=0.6032 ; \rho_{2}=0.6481 ; \rho_{3}=0.6762 ;$

$\rho_{4}=0.6573 ; \rho_{5}=0.5647$.

From the above grey relational coefficient result, one can see that $\rho_{3}=\max _{1 \leq i \leq 4} \rho_{i}$. So the historical internet opinion emergency case 3 has the greatest grey relational degree to the current unknown emergency problem, so this source case 3 is the nearest one to the current unexpected internet opinion emergency. That is to say, the historical emergency 3 is the most matching case in all the candidate emergencies stored in the designed internet opinion emergency system case base. The solution of source case $x_{3}$ is the optimal resolution of the new occurred emergency. Thus, emergency management decision-makers can adjust or modify the warning decision solution of historical internet sentiment emer- gency $x_{3}$ to facilitate the urgent decision for the current unknown internet opinion emergency. And according to the warning degree obtained by this warning decision system, the government department can take the corresponding emergency response and coordinate all kinds of emergency sources or facilities among different municipal zones to avoid or decrease the risk loss of the current unexpected internet opinion emergency.

\section{CONCLUSION}

The paper has designed a new warning decision system mechanism for uncertain internet public opinion emergency by using the fuzzy grey relational retrieval method and the invented software tools in some patents. It can improve the efficiency of emergency decision. Furthermore, we will try to develop a hybrid retrieval algorithm in the case reasoning process to enhance the flexibility and practicability of internet public opinion emergency risk forecasting system.

\section{CONFLICT OF INTEREST}

The authors confirm that this article content has no conflict of interest.

\section{ACKNOWLEDGEMENTS}

This work is supported in part by the National Social Science Fund of China (13CGL130), the Natural Science Foundation of Guangdong Province (S2013010013050, S2012040007184, S2012010010570), the National Statistical Science Research Planning Project of China (2012LY159), the National Natural Science Foundation (61273118, $61202271,61263014)$, the Scientific and Technological Innovation Project of Guangdong Province Colleges Subject Construction Special Fund (2013KJCX0069), the Humanities and Social Sciences Research Youth Foundation of Ministry of Education of China (12YJCZH281, 13YJCZH258), The High-level Talents Project in Guangdong Province (GWTP-GC-2014-03).

\section{ABOUT THE AUTHORS}

First Author Qiansheng Zhang, Professor of Guangdong University of Foreign Studies. His major is emergency management and decision. 26 papers received by SCI or EI.

Second Author Bailin Xie, $\mathrm{PhD}$ of Computer engineering in Zhongshan University. His major is internet safety detection.

Third Author Xinmeng Zhang is an associate professor of Guangdong University of Foreign Studies.

\section{REFERENCES}

[1] R.X. Zeng, "Construction of network public sentiment emergency early warning index system," Information Studies: Theory and Application, vol. 33, no.1, pp. 77-80, 2010.

[2] C. Lin, B. C. Li and J. Wang, "Network public sentiment early warning method based on fuzzy reasoning," Journal of Information Engineering University, vol. 12, no.1, pp. 72-76, 2011.

[3] Y. T. Li and Y. Liu, "Study on the grey early warning evaluation of network public sentiment," Journal of Intelligence, vol. 30, no.4, pp. 24-27, 2011. 
[4] Peking University Fangzheng intelligent public opinion aided decision support system, [Online] Available from: www.founderrd. com/2006-03/16.

[5] Goonie internet public opinion supervision analysis system, [Online] Available from: www. goonie.cn/ 2008/ products// 01/content3.html.

[6] J.H. Huang, W.Yao and Z.B. Lei, "Research on intelligent earlywarning support system for enterprise finance crisis based on case reasoning," Systems Engineering-Theory \& Practice, vol.12, no. 12, pp. 46-52, 2003.

[7] H. M. Luk, P. E. Coates, A. S. Deo, S. M. Downs, B. A. Friesen, C. A. Nies and A. K. Pathria. U.S. Patent 7, 813,944. 2010.

[8] S. M. Zoldi, L. Wang, L. Sun and S. G. Wu. U.S. Patent 7, 945, 515. 2010.

[9] Q.Y.Zhong, S. Guo, X.Ye and Y.G. Zhang, " Case representation and retrieving method for emergency aided decision," Journal of DaLian University of Technology, vol. 51, no.1, pp. 137-142, 2011.

[10] Y.J. Zhang, Q.Y. Zhong, X.Ye and X.F.Qu, "Research on method of emergency aid decision making based on CBR," Application Research of Computers, vol. 26,no. 4, pp.1412-1415, 2009.

[11] X.L. Liu, B. J. Tang and W.H. Qiu, "A search model for enterprise crisis early warning case based on gray association," China Soft Science, vol. 8, pp. 152-160, 2007.

[12] T. J. Crook. U.S. Patent 8, 001, 597. 2011.

[13] K. Roller, W. Johnson and P. I. Handerha. U.S. Patent 7, 953, 695. 2011.

[14] G. J. Sullivan, H. Geraldine, E. Rosario, M. S. Sossi, C. Ralph and J. Duchnowski. U.S. Patent 7, 895, 139. 2011.
[15] S. Kumar, E. D. Chow and M. Momma. U.S. Patent 7, 672, 865 2010.

[16] Y.W. Zhang, J.Y. Qi, B.X. Fang and Y. X. Li, "Research on the index system of public opinion on internet for unexpected emergency," Journal of Information Science, vol. 29, no. 9,pp. 14181424, 2011.

[17] J. L. Deng, "Control problems of grey systems," Systems and Controls Letters, vol. 5, pp. 288-294, 1982.

[18] D.Y. Chang, "Applications of the extent analysis method on fuzzy AHP, " European Journal of Operational Research, vol. 95, no.3, pp. 649-655, 1996.

[19] J. J. Zhang, D.S. Wu and D. L. Olson, "The method of grey related analysis to multiple attribute decision problems with interval numbers," Mathematical and Computer Modeling, vol. 42, pp. 991-998, 2005

[20] L. Lin, X. H. Yuan and Z.Q. Xia, "Multicriteria fuzzy decisionmaking models based on intuitionistic fuzzy sets, "Journal of Computer and Systems, vol. 73, pp. 84-88, 2007.

[21] F.E. Boran, S. Genc, M. Kurt and D. Akay, "A multi-criteria intuitioni stic fuzzy group decision making for supplier selection with Toposis method," Expert Systems with Applications, vol. 36, pp. 11363-11368, 2009.

[22] S.H. Wei and S.M. Chen, "A new approach for fuzzy risk analysis based on similarity measures of generalized fuzzy numbers," $E x$ pert Systems with Applications, vol. 36, no.2, pp. 589-598, 2009.

[23] C. H. Hsieh and S. H.Chen, "Similarity of generalized fuzzy numbers with graded mean integration representation," in Proceedings of the 8th International Fuzzy Systems Association World Congress, 1999 , vol. 2 , pp. 551-555

Received: September 22, 2014

Revised: November 30, 2014

Accepted: December 02,2014

(C) Zhang et al.; Licensee Bentham Open.

This is an open access article licensed under the terms of the Creative Commons Attribution Non-Commercial License (http://creativecommons.org/licenses/by-nc/3.0/) which permits unrestricted, non-commercial use, distribution and reproduction in any medium, provided the work is properly cited. 